\title{
LA EDUCACIÓN Y EL APORTE DE LAS NORMALES EN NARIÑO
}

\author{
JOSÉ OLIDEN MUÑOZ BRAVO
}

Estudiante del Doctorando en Ciencias de la Educación de la Universidad de Nariño - RUDECOLOMBIA. Docente de la Escuela Normal Superior del Mayo, en La Cruz - Nariño.

Miembro del Grupo de Investigación en Educación y Pedagogía -GIDEP- de la Universidad de Nariño.

\section{RESUMEN}

Las normales en Nariño han cumplido un gran papel en cuanto a la formación de los futuros maestros, quienes a través de la práctica pedagógica han formado los cimientos de una educación que ha contribuido con el seguimiento en valores de las nuevas generaciones de jóvenes dispuestos a luchar por una causa común, la cual dignifican en la enseñanza.

Palabras Clave: Educación, Normales, Maestros, Formación.

\section{ABSTRACT}

The normals in Nariño have played a great role in the training of future teachers, who through pedagogical practice have formed the foundations of an education that has contributed to the monitoring of the values of the new generations of young people willing to fight for a common cause, which they dignify in teaching.

Key words: Education, Normal, Teachers, Training. 
Al hablar del papel realizado por las normales de Nariño, se tendría que realizar una evaluación del trabajo organizado por los maestros para apoyar un sinnúmero de actividades que fortalezcan y demuestren los logros alcanzados en los comienzos del siglo XX.

Como bien es sabido, las normales han sido gestoras de transformaciones educativas y pedagógicas, las cuales han servido de base para formar un maestro de calidad que esté muy comprometido con la enseñanza y el fortalecimiento de la comunidad.

Los maestros son constructores de experiencias pedagógicas, capaces de diseñar sus propias escuelas, crear métodos de pensamiento, escribir; y reflexionar sobre su quehacer en el aula, pueden ser investigadores tanto de sus propias prácticas, de sus memorias de saber. Las Escuelas Normales, tienen que formar un maestro de un cambio generacional en el campo epistemológico de la educación y de la investigación.

Estas instituciones van a ser las que se apropien de la actitud para crear potencialidades y medios que en un determinado plazo le permitan, conjuntamente con las universidades, formar un nuevo maestro que esté en condiciones de aportar esos conocimientos, en el análisis que los niños y jóvenes esperan para que sus sueños sean realidades.

Las Normales de este siglo, deben orientar en cuanto a la enseñanza, la pedagogía activa y la investigación, lo que hacen diariamente, tienen como reto guiar a los futuros maestros tomando como fundamento las dimensiones: Ética y pedagogía.
Las normales buscan fortalecer la pedagogía como elemento esencial de la formación del maestro, producto de la reflexión permanente, de la crítica social y del pensamiento complejo, hacer de la educación una práctica social centrada en la proyección del aprendizaje.

Las normales en Nariño han cumplido un gran papel en cuanto a la formación de los futuros maestros, quienes a través de la práctica pedagógica han formado los cimientos de una educación que ha contribuido con el seguimiento en valores de las nuevas generaciones de jóvenes dispuestos a luchar por una causa común, la cual dignifican en la enseñanza.

La Ley 39 de 1903, producto de la reforma educativa impulsada por el ministro de Instrucción Pública Antonio José Uribe a comienzos de siglo, favorecía la idea de organizar en Pasto dos instituciones para la formación de maestros. La norma dispuso la existencia en cada una de las capitales de los departamentos de una escuela normal para varones y otra para mujeres, costeadas por la Nación é invigiladas por el respectivo Gobierno departamental ${ }^{1}$.

Sin embargo, en 1905 la política de Rafael Reyes dio un giro que dificultó la creación de la Normal masculina. El Presidente Reyes dejó la formación de maestros para las escuelas de niños bajo la dirección moral y pedagógica de los Hermanos Cristianos ${ }^{2}$. Para tal efecto se fundó en Bogotá la Escuela Normal Central de Institutores ${ }^{3}$. En los departamentos a cargo del presupuesto nacio-

1. Ley 39 de 1903 (26 de octubre). Artículo 23. En: Diario Oficial.

2. Decreto Número 1244 de 1905 (21 de octubre).

3. Decreto Número 1244 de 1905 (21 de octubre). Artículo 60. 
nal quedaron únicamente las Escuelas Normales de mujeres de Cartagena, Medellín, Popayán, Pasto, Bucaramanga, Tunja y Bogotá4.

En las nuevas condiciones, a pesar del interés y la presión de algunos ciudadanos porque se abriera la institución para los jóvenes, el gobierno departamental no tuvo otra alternativa sino la de organizar en primera instancia la Escuela Normal de Institutoras, el proyecto de la Normal de Varones quedó aplazado hasta 1911.

Ante una realidad tan evidente como era la imposibilidad de crear esta Normal en 1905, el gobierno departamental y en especial el Director de Instrucción Pública don Enrique Muñoz decidieron apoyar a jóvenes que quisieron ingresar en la Normal Central de Bogotá para que, cuando las condiciones lo permitieran, hicieran parte del equipo docente encargado de preparar a los maestros en la región. Efectivamente, bajo esta perspectiva estudiaron con los Hermanos Cristianos los señores Clodomiro Díaz del Castillo y Delfín Reinel.

\section{Historia de la Normal Superior de Pasto, Nariño}

La presencia en el Congreso Nacional de personajes como don Ildefonso Díaz del Castillo favoreció la expedición de la Ley 7a. de 1911 sobre la cual se firmó el Decreto departamental 422 del 7 de noviembre de 1911 que permitió la apertura de la Escuela Normal de Varones de Pasto el 23 de noviembre de 1911. En el momento era gobernador del Departamento el general Gustavo S. Guerrero

4. Para Reyes los Hermanos Cristianos garantizaban una enseñanza según lo dispuesto por el Vaticano, además tenían experiencia en la formación de maestros y en la enseñanza técnica. En: HELG, Aline. La educación en Colombia 1918-1957. Bogotá, Fondo Editorial Cerec, 1987, p. 78. quien nombró a don Enrique Muñoz como primer Director de la Normal, de Subdirector a Clodomiro Díaz del Castillo, de Director de la Escuela Anexa a Delfín Reinel, como celadores-profesores a Roberto Patiño y Mario A. Guerrero. La Institución inició formalmente clases el 8 de enero de $1912^{5}$.

La Normal de Varones respondió a los principios morales y religiosos que orientaron el proyecto educativo conservador. Se trataba de formar, como ya se indicó, un maestro eminentemente católico, modelo de vida para la sociedad. La enseñanza de la doctrina católica fue obligatoria, tal y como lo señalaban los planes de estudio, igualmente tenían que asistir a misa los domingos y días de fiesta religiosa, confesarse y comulgar6. Aspectos en los cuales no difería de las demás instituciones educativas del país.

Teniendo en cuenta el Reglamento de las Escuelas Normales, vigente para la Normal durante el período, los estudiantes debían estar bien presentados, el doctor Muñoz quiso establecer uniforme pero finalmente tuvo que desechar la idea, no podían consumir licor, ni practicar juegos de azar, ni entablar discusiones políticas ${ }^{7}$.

En la Escuela Normal de Institutoras de Nariño las estudiantes fueron preparadas, además de las anteriores, en: costura, bordados, tejidos, confección de ropa para niños, modistería y floristería, ${ }^{\mathbf{8}}$ más adelante también en culinaria.

5. BUENDÍA N., Jorge. La Escuela Normal de Varones de Pasto. Pasto, Imprenta Departamental, 1974, p. 8.

6. Según lo establecido en el Concordato con la Santa Sede.

7. Reglamento para las Escuelas Normales. Artículo 27. Numerales 4, 7, 20.

8. Acta de Visita practicada en la Escuela Normal de Institutoras, en diciembre de 1919. En: Registro de Instrucción Pública, Números 2, 3 y 4, Pasto, Enero de 1920, p. 120. 
Los estudios en la Normal de Varones se iniciaron con el Plan de Uribe pero como en 1912 se presentó el primer cambio, les correspondió complementar con lo dispuesto en el nuevo Plan. En la misma forma que la Normal de Institutoras, durante el período los estudios estuvieron orientados con el Plan de 1913. Este Plan aumentó los estudios a cinco años, sin dejar el concepto básico de maestros prácticos más que pedagogos eruditos, con la reforma se aprobaron asignaturas con una visión más amplia en cuanto a la formación del maestro que la diseñada por el doctor Uribe, introdujeron nuevamente Física, Química, Francés, Lógica y Retórica9.

La enseñanza para la formación práctica estuvo centrada en las actividades que desarrollaron en la huerta escolar, organizada en predios de la Institución, allí los estudiantes aprendían técnicas sencillas para la producción agrícola de los cultivos propios de la región.

Para el desarrollo de la práctica pedagógica la Normal contó desde sus inicios con una Escuela Anexa a cargo de un Director quien también tenía como funciones la enseñanza Teórica de la Pedagogía y la supervisión de las clases de los alumnos-maestros, así como de los demás ejercicios propios del campo de la Pedagogía práctica.

A partir de 1926 y por iniciativa del último Director de la Normal don Jorge Buendía Narváez ${ }^{\mathbf{1 0}}$, la preparación pedagógica de los estudiantes se complementó con la introducción de asig-

9. HERNÁNDEZ VEGA, Gabriela. Formación de maestros en el departamento de Nariño. 2004.

10. Egresado en la primera promoción de la Normal, profesor de Estado de Chile, Director de Instrucción Pública del Departamento, cargo que le permitió ser Presidente del Consejo Superior de la Universidad de Nariño, Director de la Escuela Normal de Varones desde el 23 de noviembre de 1923 hasta el 11 de enero de 1930. naturas como Historia de la Educación y Psicología Pedagógica.

A partir de 1924, siendo director don Jorge Buendía e inspirados en el Gimnasio Moderno realizaron excursiones a diferentes lugares de la región las cuales tenían como eje un Centro de Interés que respondía a hechos o lugares destacados según el sitio de la excursión. Cada excursión estaba a cargo de uno de los profesores quien preparaba una conferencia alusiva al Centro de Interés, con este enfoque llevaron a cabo viajes a: Campo de la Batalla de Bomboná, a las Provincias de Obando y Túquerres, a las Provincias del Juanambú y La Cruz, a Tumaco en la Costa Pacífica y al oriente del Departamento.

El profesor Buendía concibió las excursiones como un medio pedagógico que permitían la satisfacción de múltiples necesidades educativas: fortalecimiento del cuerpo, enriquecimiento de las nociones científicas, geográficas, históricas, y servían para complementar la formación moral ${ }^{\mathbf{1 1}}$. Para él, cuando los profesores enseñaban las riquezas de una tierra que ellos habían podido conocer y con los sentidos aún empapados por imágenes del lugar seguramente, sus enseñanzas tendrán un interés supremo, su voz será más cálida y, por ende, los frutos serán óptimos ${ }^{\mathbf{1 2}}$.

\section{Historia de la Normal Superior del Mayo, La Cruz - Nariño}

En el año de 1938 la Asamblea de Nariño, creó en la ciudad de La Cruz, el Colegio Departamental, Liceo Mayo, que en 1945 serviría de base para que

11. BUENDÍA, Jorge. Extensión cultural de la Escuela Normal de Institutores de Pasto 1923-1929. Pasto, Imprenta de Díaz del Castillo y Cía, p. 320.

12. BUENDÍA, Extensión... Op. Cit. p. 321. 
la Nación creara la Normal Rural de Señoritas que en 1956, la Secretaría de Educación del Departamento resolvió convertir en Normal para Varones "Rafael Reyes"; y más tarde, en el año de 1958 por petición de la ciudadanía, la Junta Militar de Gobierno dictó el Decreto-Ley No.0238 de 11 de julio nacionalizando dicha Institución.

El Ministerio de Educación dictó en 1963 el Decreto 1955 mediante el cual se terminan las Normales Rurales, pero en 1965, un movimiento cívico, logra la superiorización de esta Institución, que promocionó a sus primeros Maestros con seis años de estudio en julio de $1967^{13}$.

Históricamente La Cruz ha tenido una tradicional vocación pedagógica, comprobable en los anales de Nariño y del país; ésta es razón suficiente para merecer el título de "Ciudad Maestra", cuna de cultura, en donde se brinda a través de su Normal y otras instituciones educativas una sólida formación integral de la que son testigos quienes de alguna manera han recibido su benéfica influencia.

En el año lectivo 1968-1969 y considerado como un acontecimiento histórico de la población, se matricularon en los dos años del Ciclo Profesional Normalista las primeras mujeres, y en 1973 se implementó la educación mixta. Para todos los niveles. Hoy en la Normal se piensa profundamente sobre las bondades o perjuicios de la coeducación, pues desafortunadamente se ha perdido el respeto entre los jóvenes y las condiciones de género no representan un mayor esfuerzo para luchar por una sana y armoniosa convivencia.

13. PEI. Escuela Normal Superior del Mayo, La Cruz, Nariño.

\section{Historia de la Normal Superior Sagrado} Corazón de Jesús, San Pablo, Nariño

El municipio cuenta con varios centros educativos, entre los cuales se levanta honrosa la Escuela Normal Superior "Sagrado Corazón de Jesús", institución centenaria fundada en el año de 1909, gracias al párroco Aquilino Zambrano, los padres de familia, y las hermanas Bethlemitas, quienes se encargaron de la educación de los pobladores. En el año de 1937, se le otorgó licencia de funcionamiento como "Escuela Normal Rural Sagrado Corazón de Jesús" para señoritas, con 4 grados, licencia que perduró hasta el año de 1957. En el año 1962 se organizó el Ciclo Básico de educación Media y en 1967 se aprueba el ciclo profesional normalista. El 13 de febrero de 1974, fue anexada la escuela Urbana de niñas de San Pablo a la Normal departamental mixta "Sagrado Corazón de Jesús". En 1975 fueron aprobados los estudios de básica primaria, secundaria y media vocacional, donde los egresados eran bachilleres pedagógicos. En 1981 se aprueban los estudios correspondientes al nivel preescolar ${ }^{14}$.

En la actualidad cuenta con 990 estudiantes procedentes del sector rural del municipio, del casco urbano y del sur del Cauca. El personal docente es licenciado, y en su mayoría con estudios de postgrado. El personal administrativo $\mathrm{y}$ de servicios generales es trabajador y demuestran buenas relaciones interpersonales. Los padres de familia demuestran compromiso y participación con la institución. Las relaciones entre los diferentes estamentos son buenas porque se convive en un ambiente de cordialidad, camaradería y respeto.

14. PEI. Institución Educativa Sagrado Corazón de Jesús, San Pablo, Nariño. 
Desde su acreditación previa, moviliza la comunidad educativa, para generar, desarrollar y gestionar procesos que permitan demostrar la calidad y desempeño en la formación del nuevo maestro. Por lo tanto, la institución pone en marcha el Proyecto Educativo, como alternativa eficaz para lograr el desarrollo de la región y del país.

\section{Historia de la Normal Superior San Carlos, La Unión, Nariño}

La Institución Educativa Escuela Normal Superior San Carlos de la Unión Nariño, fue fundada por la Beata Caridad Brader, el 19 de Septiembre de 1915, como respuesta a la necesidad de formar maestros para atender los requerimientos educativos de las familias y especialmente de la niñez del Norte de Nariño. Su fundadora de nacionalidad suiza, también fundadora de la congregación de las Hermanas Franciscanas de María Inmaculada, hace posible el asentamiento de una casa de las religiosas en esta región y en adelante orientan hasta la fecha esta Institución desde una concepción pedagógica y cristiana. La Fundadora asigna como patrono a San Carlos Borromeo.

En el año 1957-1958, se logró la Nacionalización del Colegio, con la autorización para iniciar orientación Normalista. El 27 de abril de 1958, se celebró el contrato de nacionalización y se fijó como tiempo de duración de los mismos 20 años. El 18 de Julio de 1962, la Escuela Normal de Señoritas San Carlos, tuvo la satisfacción de proclamar como alumnas maestras a 14 señoritas, siendo la primera promoción de Normalistas Superiores.
En el año de 1972 fueron aprobados los estudios de Enseñanza Primaria, Ciclo Básico de Enseñanza Media y Ciclo Profesional Normalista. Posteriormente en el año de 1979 se efectuó otra visita de supervisión y la institución fue aprobada, adoptando un plan de estudios ordenado por el Decreto 080 de 1974 y la Resolución 4785 del 9 de junio del mismo año, reglamentando la formación normalista ${ }^{15}$.

En 1984, con base en el Decreto 2647 mediante el cual se reglamenta la Institución, se reestructuró el proyecto de práctica docente, y a partir de 1987, surgieron cambios significativos en el proceso de formación del futuro maestro.

Estos cambios hicieron que en 1990 se originara el proceso de reforma de la propuesta de formación de maestros, y al interior de la institución se geste un movimiento pedagógico, razón por la cual se recurre a capacitación proporcionada por profesionales reconocidos en el campo educativo, a pesar de no existir aún la reglamentación de los proyectos educativos institucionales, la Escuela Normal ya traía algunos avances al respecto, lo cual facilitó la adaptación al proceso de reestructuración que a partir de 1996-1997 se debería asumir y que condujo a la Acreditación Previa en 1999, a la Acreditación de Calidad y Desarrollo en el año 2003, dando grandes pasos en la resignificación de las prácticas pedagógicas y administrativas de la Institución.

15. PEI Escuela Normal Superior San Carlos de La Unión, Nariño 
Historia de la Normal Superior Pío XII de Pupiales, Nariño

Está ubicada en Pupiales Nariño, en 1904 se fundó el Convento de la Inmaculada Concepción, bajo la dirección de la reverenda madre Caridad, en 1914 se fundó un colegio de señoritas bajo el título La Inmaculada Concepción.

El 14 de diciembre de 1923 la región sufrió un temblor y el edifico se vino abajo pero fue reconstruida.

En 1929 se celebraron las bodas de plata y 1954 se celebraron las bodas de oro.

Se crea una Normal para señoritas y mediante Decreto No. 108 se creó la Normal de Señoritas Pío XII, en noviembre 22 se celebraron las bodas de diamante donde acudieron muchas personalidades del municipio ${ }^{16}$.

A comienzos de 2004 se celebraron cien años de existencia franciscana en nuestro municipio y cien años de existencia en nuestra institución.

Por medio de reflexiones se promueve la vivencia de valores franciscanos, los principios institucionales se fundamentan en la pedagogía de la Madre Caridad y la espiritualidad franciscana.

\section{Historia de la Normal Superior La Inmaculada de Barbacoas, Nariño}

Aprobación oficial de la Escuela Normal Rural de Señoritas la "Inmaculada" de Barbacoas, con Resolución No. 02902, del 30 de junio de 1958.

16. PEI, Institución Educativa Normal Pio XII de Pupiales, Nariño.
En 1958 visita a la Normal el político Luis Avelino Pérez, quien consiguió la aprobación de la escuela como establecimiento educativo nacional.

Según resolución 3905 del 25 de agosto 1959, se aprueba los estudios normalistas en todos los cursos.

Las carmelitas misioneras en Barbacoas, a lo largo de su historia han tenido presencia apostólica en el campo educativo en la Normal Superior "La Inmaculada" (1954-1978).

El 19 de junio de 1978 Aprobación del Consejo General del retiro de las hermanas de la Normal superior "La Inmaculada".

Uno de los propósitos de la Escuela Normal Superior la Inmaculada de Barbacoas es formar maestros investigadores. En este intento, la investigación con enfoque pedagógico es uno de los ejes trasversales que enmarca el quehacer cotidiano. La propuesta es que los maestros en formación participen a través de su práctica docente en la ejecución de proyectos pedagógicos que se implementan en la Normal. Para ello resulta necesario e importante que estos realicen sus labores en cada uno de los grados de preescolar y básica primaria.

El proceso de formación se desarrolla en cuatro etapas:

Grado cero a sexto: periodo básico de formación integral humana.

Grado séptimo a noveno: periodo de transición e iniciación pedagógica.

Grado diez a once: periodo de formación media académica con profundi- 
zación en el campo de la educación y formación pedagógica.

Ciclo complementario: se aborda la formación del maestro, profundizando en los campos pedagógicos, disciplinar, científico, investigativo y de valores.

La Normal Superior de Barbacoas le entrega a la sociedad colombiana egresados con Títulos de Normalista Superior Etnoeducador, previa introducción de la etnoeducación afrocolombiana desde el Preescolar hasta la Formación Complementaria, como el eje que permite el desarrollo de la formación en la institución.

La formación complementaria para maestros se da con la expedición del decreto 4790 de diciembre de $2009^{17}$, que reorienta el quehacer del programa de formación complementaria de las Escuelas Normales Superiores de Nariño. Se busca las condiciones básicas de calidad que conllevan a la formación de profesionales coherentes con la nueva sociedad.

El maestro en formación es un constructor de realidades, como un ser que crece en la medida en que interactúa y colabora con otros; un ser capaz de aprender en donde pueda realizarse en la propia realidad que construye.

Por lo tanto el maestro en formación será un maestro orientador de procesos y propiciador de condiciones problematizantes que desarrolla su capacidad intelectual, emocional, social y física. Un maestro que se reconozca a sí mismo

17. Decreto 4790 de diciembre de 2009, emanado del Ministerio de Educación Nacional. como sujeto y objeto de transformación, y que reconozca su entorno inmediato.

Ahora en Colombia los procesos de acreditación de los programas de formación de maestros vienen siendo implementados por el MEN-ICFES, para las facultades de educación y Escuelas Normales Superiores cada cierto período de tiempo, donde se fijan criterios básicos que deben cumplirse para la legalización del programa. En lo que respecta a Escuelas Normales le corresponde al Consejo de Acreditación de las Escuelas Normales Superiores (Caens) realizar el seguimiento y acreditación previo cumplimiento de los requisitos exigidos. Es de anotar que cuando se autorizan programas de formación docente para una o dos promociones esta directiva no es aplicable, por lo cual no hay seguimiento, por las condiciones de descentramiento.

Según documento del Caens, denominado Formación de Maestros - elementos para el debate, sostiene:

Si se mira la génesis de las escuelas normales y su evolución e incluso el desarrollo mismo de las facultades de educación- es fácil encontrarse con el hecho de que ambas han centrado su labor de formación en la reproducción de modelos de enseñanza que le dan preeminencia a una sola de las múltiples y variadas tareas de educador: la de dar o impartir clase. Con ello se restringía la educación al ámbito del salón de clase y, además, se asumía que la identidad del maestro estaba circunscrita a la de simple instructor; es decir, como transmisor de un saber objetivado con la intención de que éste fuera retenido o repetido por parte del aprendiz o como aquella persona que sólo estaba en condiciones de repetir contenidos, recibir y seguir instrucciones claramente planificadas 
por otras personas, muchas veces alejadas de la cotidianidad de la escuela. (Caens, 2000)

De otra parte Carvajal Ortiz y otro, manifiestan:

...Las Escuelas Normales de nuestro país y específicamente las de nuestra región, están llamados a ser, fortalecer y mantener el bastión que permita entrever esa utópica posibilidad, la de crear seres humanos críticos y propositivos, éticamente competentes y capaces de transmutar lo que aun hoy, quienes cavilamos en estas situaciones no hemos logrado transformar (Carvajal Ortiz et al, 2012, p. 160).

Según estos autores:

El papel del $\mathrm{PFC}^{18}$ y la razón de ser, de dichas estructuras es crear y darle al maestro en formación las herramientas necesarias para ir entreviendo el escenario del ser docente; escenario donde el maestro en formación entrará a hacer parte esencial, ya no como un simple espectador sino como un protagonista de un proceso de cambio social, el cual nace en la utopía educativa que todo buen maestro persigue, la de pensar su escuela a partir de la que tiene para acercarse a la que sueña y la sociedad requiere. Se convierte así, el P.F.C en una posibilidad dual, ya que no solo es génesis de los futuros maestros que ingresan a él, sino también fuente esperanzadora para nuestra sociedad tan necesitada y urgida de seres humanos vivos, con deseos de proponer y transformar el desorden que brutal o ingenuamente, hemos venido causando las generaciones que les hemos antecedido (Carvajal Ortiz et al, 2012, p. 161).

18. PFC. Programa de formación complementaria.

\section{CONCLUSIONES}

Las normales en Nariño, cada día demuestran que tienen un gran compromiso con la sociedad y con el país, para entregar docentes con un alto valor intelectual, afectivo y basado en valores que contribuyan a la orientación de la niñez y la juventud.

Las normales en Nariño, en la actualidad tienen un gran liderazgo en cuanto a la profundización pedagógica e investigativa, donde sus estudiantes adquieren autonomía para acompañar procesos innovadores que mejoren la calidad educativa.

Hoy las normales compiten sanamente en demostrar todo su componente humano para formar maestros con vocación que entreguen sus conocimiento y su práctica pedagógica en bien de las comunidades más débiles y necesitadas del país.

Los maestros egresados de las normales en Nariño tienen una gran acogida en diferentes zonas del país, demostrando con ello la tenacidad y la entrega a su labor pedagógica, donde sus niños les demuestran afecto y responsabilidad para adquirir sus compromisos y retos personales. 


\section{BIBLIOGRAFÍA}

BUENDÍA N., Jorge. La Escuela Normal de Varones de Pasto. Pasto, Imprenta Departamental, 1974.

BUENDÍA, Jorge. Extensión cultural de la Escuela Normal de Institutores de Pasto 1923-1929. Pasto, Imprenta de Díaz del Castillo y Cía.

CARVAJAL, L. \& CERTUCHE, L. C. (2012). Reflexiones acerca de la formación de formadores. Colección: Pedagogía y Ciencia, No. 1. Primer encuentro de Pedagogía y Ciencia. Cauca: Editorial Normal Santa Clara.

CONSEJO NACIONAL DE ACREDITACIÓN (1998). Criterios y procedimientos para la acreditación previa de los programas académicos de pregrado y especialización en educación. Santafé de Bogotá: Sistema Nacional de Acreditación.

Decreto 4790 de diciembre de 2009, emanado del Ministerio de Educación Nacional.

Decreto Número 1244 de 1905 (21 de octubre).

Decreto Número 1244 de 1905 (21 de octubre). Artículo 60.

Ley 39 de 1903 (26 de octubre). Artículo 12. En: Diario Oficial, Número Año 1903.

MEN. (2011). Perfil del Normalista Superior. Recuperado de http://www.mineducacion.gov.co/1621/articles239511 archivo.pdf Quintero, M., \& Ruiz, A. (2005).

PEl, Escuela Normal Superior San Carlos de La Unión, Nariño.

PEl, Institución Educativa Normal Pio XII de Pupiales, Nariño.

PEl. Escuela Normal Superior del Mayo de La Cruz, Nariño

PEl. Institución Educativa Sagrado Corazón de Jesús de San Pablo, Nariño. 